The FASEB Journal express article 10.1096/fj.02-0574fje. Published online January 2, 2003.

\title{
Intravascular tissue factor initiates coagulation via circulating microvesicles and platelets
}

Ingrid Müller,* Antje Klocke, ${ }^{*}$ Meike Alex, ${ }^{*}$ Matthias Kotzsch, ${ }^{\dagger}$ Thomas Luther, ${ }^{\dagger}$ Eberhard Morgenstern, ${ }^{\star}$ Susanne Zieseniss, ${ }^{*}$ Stefan Zahler, ${ }^{*}$ Klaus Preissner, ${ }^{\S}$ and Bernd Engelmann*ף

*Physiologisches Institut and ${ }^{\uparrow}$ Vaskuläre Biologie und Hämostase, Institut für Klinische Chemie, Ludwig-Maximilians-Universität München, 81377 München; †'Institut für Pathologie,

Technische Universität Dresden, 01307 Dresden; ${ }^{*}$ Medizinische Biologie, Universität des

Saarlandes, Campus Homburg, 66421 Homburg; and ${ }^{\S}$ Institut für Biochemie, Universität

Giessen, 35392 Giessen, Germany

Corresponding author: Bernd Engelmann, Institut für Klinische Chemie, Vaskuläre Biologie und Hämostase, Klinikum der Universität München, Marchioninistr. 15, 81377 München, Germany.

E-mail: Bernd.Engelmann@klch.med.uni-muenchen.de

\section{ABSTRACT}

Although tissue factor (TF), the principial initiator of physiological coagulation and pathological thrombosis, has recently been proposed to be present in human blood, the functional significance and location of the intravascular TF is unknown. In the plasma portion of blood, we found TF to be mainly associated with circulating microvesicles. By cell sorting with the specific marker CD42b, platelet-derived microvesicles were identified as a major location of the plasma TF. This was confirmed by the presence of full-length TF in microvesicles acutely shedded from the activated platelets. TF was observed to be stored in the $\alpha$-granules and the open canalicular system of resting platelets and to be exposed on the cell surface after platelet activation. Functional competence of the blood-based TF was enabled when the microvesicles and platelets adhered to neutrophils, as mediated by P-selectin and neutrophil counterreceptor (PSGL-1, CD18 integrins) interactions. Moreover, neutrophil-secreted oxygen radical species supported the intravascular TF activity. The pools of platelet and microvesicle TF contributed additively and to a comparable extent to the overall blood TF activity, indicating a substantial participation of the microvesicle TF. Our results introduce a new concept of TF-mediated coagulation crucially dependent on TF associated with microvesicles and activated platelets, which principally enables the entire coagulation system to proceed on a restricted cell surface.

Key words: lipopolysaccharide - platelet rich plasma - superoxide dismutase - catalase • microparticles

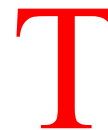

Two principal events that are initiated after disrupture of the endothelial barrier are thought to mark the initiation of hemostasis. Blood platelets adhere to subendothelial collagen providing a provisional, mechanically unstable closure of the vessel perforation. Concomitantly, the coagulation process is started. This is mainly due to the formation of an initiator complex between tissue factor (TF), an integral cell membrane protein predominantly present in the adventitial layer of the vessel wall, and the blood-based factor VII/VIIa (1). The $\mathrm{TF} /$ factor VIIa complex proteolytically activates factor $\mathrm{X}$, which, in turn, elicits the formation of 
thrombin. The TF/factor VIIa complex is likely to play a central role in the genesis of arterial and venous thrombosis $(2,3)$, leading causes of mortality in many countries. TF is present in the lipid rich core of unstable atherosclerotic plaques and may be a major determinant of the thrombogenicity of the plaques (4-6). Indeed, on rupture of the plaque, the interaction of TF with factor VIIa substantially contributes to the rapid formation of the occluding thrombus, the principal final step in the genesis of coronary ischemic disease.

Apart from its presence in the vascular wall, TF has also been detected in the blood (intravascular TF). So far, no clear conclusion has been reached about the localization and the functional meaning of the blood-based TF. In the plasma compartment, TF is present under physiological conditions and its concentration is increased in patients with unstable angina and myocardial infarction (7-9). The presence of TF in neutrophils and/or unstimulated monocytes has been a matter of debate (10-12). Prolonged activation of the monocytes with lipopolysaccharide (LPS) induces the expression of $\mathrm{TF}$ as a result of the transcriptional activation of the TF gene (13). On the other hand, collagen was shown to rapidly start coagulation in a way depending on TF being associated with the platelets (14). With the use of a promonocytic cell line stimulated with LPS, it was shown that TF can in principal be transferred onto the platelets via microparticle transfer (15). The knowledge of the mechanism of activation of the blood-based TF is of great relevance for the understanding of its role under pathological conditions as well as for potential therapeutic interventions. The results indicate a crucial role for the TF on circulating microparticles and platelets as a trigger for the overall start of coagulation.

\section{METHODS}

\section{Antibodies}

The monoclonal anti-human TF antibodies directed against extracellular epitopes of the human TF were clones VIC7, VD8, and IIID8 (16). Fluorescein-isothiocyanate (FITC)-labeled monoclonal anti-human TF antibody, phycoerythrin (PE)-labeled monoclonal anti-CD14, and anti-CD15 antibodies were obtained from Biotrend. The FITC- and PE-labeled $\operatorname{IgG}_{1}$ isoantibodies as well as the labeled anti-CD42b and anti-CD66b antibodies were purchased from Becton Dickinson. The anti-CD18 and anti-PSGL-1 antibodies were from Calbiochem, the antifactor IX antibody was from American Diagnostica, and the anti-P-selectin antibody was from Ancell.

\section{Cells}

For the isolation of platelets, venous blood from healthy volunteers (age range 23-32 yr) was drawn into sodium citrate $(0.38 \%$ final concentration) and subsequently centrifuged at $330 \mathrm{~g}$ for $15 \mathrm{~min}$. The upper two-thirds of the supernatant representing the platelet-rich plasma (PRP) were again centrifuged at $330 \mathrm{~g}$ for $10 \mathrm{~min}$, and the platelet pellet was recovered and resuspended in a buffer composed of $145 \mathrm{mM} \mathrm{NaCl}, 10 \mathrm{mM}$ Hepes, $5 \mathrm{mM} \mathrm{KCl}, 1 \mathrm{mM} \mathrm{MgCl}_{2}$, and $5 \mathrm{mM}$ glucose ( $\mathrm{pH} 7.4$; resuspension buffer). The suspensions of the isolated platelets contained $<0.05 \%$ of total leukocytes. To prepare platelet-poor plasma (PPP), PRP was centrifuged for $15 \mathrm{~min}$ at $1,000 \mathrm{~g}$ and the upper $80 \%$ of the supernatant was recovered. Subsequently, the supernatant was again centrifuged at 1,000 $\mathrm{g}$, and the resulting supernatant was used as PPP. Flow cytometric evaluation indicated that platelets were absent from the PPP. Human neutrophils were prepared 
by incubation of freshly obtained buffy coats with microbeads coupled to anti-CD15 antibodies (Miltenyi Biotec) for $15 \mathrm{~min}$ at $8^{\circ} \mathrm{C}$. The suspensions were thereafter applied onto the positive selection column, and neutrophils were eluted with the antibody buffer. The purity of the suspensions thus obtained was $90 \%$.

\section{Isolation and generation of microvesicles}

The circulating microvesicles present in the plasma compartment of human blood were isolated essentially as described before (17). Two milliliters of whole blood containing sodium citrate $(0.38 \%$ final concentration) and corn trypsin inhibitor $(30 \mu \mathrm{g} / \mathrm{ml}$; Haemochrom Diagnostica; for inhibition of the contact pathway) were centrifuged at $1,550 \mathrm{~g}$ for $20 \mathrm{~min}$. Thereafter, $250 \mu 1$ of the cell free supernatant (PPP) were centrifuged for $30 \mathrm{~min}$ at 17,570 $\mathrm{g}$. In some cases, the circulating microvesicles were subsequently washed once with PBS. When hirudin was used as anticoagulant, similar amounts of micovesicles were calculated to be present in the plasma as with citrated blood. To isolate the circulating microvesicles from larger blood volumes, $20 \mathrm{ml}$ of the anticoagulated blood were centrifuged at $330 \mathrm{~g}$ for $15 \mathrm{~min}$, and the PRP was centrifuged at $1,750 \mathrm{~g}$ for $30 \mathrm{~min}$. The supernatant thus recovered was centrifuged at $168,000 \mathrm{~g}$ for $210 \mathrm{~min}$, and the microvesicle pellet was recovered. Usually, the pellet was washed before any further analyses.

To determine the size of the microvesicles by flow cytometry, large unilamellar vesicles (LUV) of defined diameter were generated by dispersion of egg phosphatidylcholine in $150 \mathrm{mM} \mathrm{NaCl}$ and $20 \mathrm{mM}$ HEPES ( $\mathrm{pH} 7.5$ ). The suspension was extruded 15 times through membranes with a pore diameter of 100, 400, and $800 \mathrm{~nm}$ (Avestin Inc.). The sideward characteristics of the LUV thus obtained were compared with 50 -fold dilutions of the isolated microvesicle suspensions. The volume was determined corresponding to a total of 10,000 events in order to obtain an estimate for the number of microvesicles present in the suspensions. For the generation of platelet microvesicles in vitro, the isolated platelets $\left(2 \times 10^{9}\right)$ were stimulated for 15 to $120 \mathrm{~min}$ with collagen $(8 \mu \mathrm{g} / \mathrm{ml})$ and thrombin $(0.5 \mathrm{U} / \mathrm{ml})$ at $37^{\circ} \mathrm{C}$. After centrifugation at $2,240 \mathrm{~g}$, the supernatant was collected and again centrifuged for $15 \mathrm{~min}$ at $18,700 \mathrm{~g}$. Thereafter, the microvesicle pellet was recovered and taken up in the resuspension buffer. Monocytic microvesicles were prepared by stimulating the isolated monocytes $\left(5 \times 10^{6}\right)$ for up to $16 \mathrm{~h}$ with $10 \mu \mathrm{g} / \mathrm{ml} \mathrm{LPS}$ at $37^{\circ} \mathrm{C}$. To generate the neutrophil specific microvesicles, the neutrophils $\left(5 \times 10^{6}\right)$ were stimulated with $100 \mu \mathrm{M}$ fMLP for $2 \mathrm{~h}$ at $37^{\circ} \mathrm{C}$ in the presence of $2 \mathrm{mM} \mathrm{CaCl}_{2}$. The cell suspensions were centrifuged at 2,240 $\mathrm{g}$ for $15 \mathrm{~min}$, followed by centrifugation of the cell free supernatant at $168,000 \mathrm{~g}$ for $90 \mathrm{~min}$.

\section{Electron microscopy}

For the evaluation of the presence of $\mathrm{TF}$ in platelets, washed platelets were fixed by impact freezing on a metal-mirror [attachment MM 80 to the KF 80 cryofixation unit (Reichert-Jung, Vienna, Austria)], cooled with liquid nitrogen, and then freeze dried in a Leica CDF unit (Vienna, Austria). Freeze drying was started at $-140^{\circ} \mathrm{C}$. At $10^{-4} \mathrm{mbar}$, the samples were dried for $225 \mathrm{~h}$ under increasing temperatures up to $0^{\circ} \mathrm{C}$ and embedded in Spurr's medium. Serial sections were prepared from these samples. Immunolabeling for human TF on ultrathin serial sections was performed using the antibodies VIC7 and VD8 $(20 \mu \mathrm{g} / \mathrm{ml}$ each). After being washed in PBS containing $0.05 \mathrm{M}$ glycine, labeling was performed for $1 \mathrm{~h}$ at $20^{\circ} \mathrm{C}$. The secondary labeling with 
goat antibodies ( $6 \mathrm{~nm}$ gold conjugated to anti-mouse $\mathrm{IgG}$ ) was performed for $60 \mathrm{~min}$ at $20^{\circ} \mathrm{C}$. Control labelings were performed without the primary antibodies. Finally, the sections were stained with uranyl acetate and lead citrate and examined in an EM 109 microscope (Carl Zeiss, Oberkochen/Germany).

For the detection of $\mathrm{TF}$ in the whole blood, blood samples (anticoagulated by citrate) were stimulated for $10 \mathrm{~min}$ at $37^{\circ} \mathrm{C}$ with collagen $(12 \mu \mathrm{g} / \mathrm{ml}$, type I, Nycomed) and incubated for 30 min at room temperature with the anti-TF antibodies (VD8 and VIC7; $10 \mu \mathrm{g} / \mathrm{ml}$ each). The samples were treated with lysing solution, fixed, washed, and incubated for $1 \mathrm{~h}$ at $37^{\circ} \mathrm{C}$ with gold-labeled goat anti-mouse IgG. Ultrathin sections were mounted on nickel grids. To visualize the isolated circulating microvesicles, suspensions of the isolated microvesicles were fixed by adding an equal volume of 2\% paraformaldehyde in PBS. Twenty microliters of this suspension were adsorbed for 30 min to Pioloform-coated grids by floating the grids on drops placed on parafilm. Grids with adhering vesicles were rinsed with water, stained with $1 \%$ uranyl acetate for 2 min, and examined by electron microscopy.

\section{Confocal laser microscopy}

The association of the mepacrine labeled circulating microvesicles with the isolated neutrophils was evaluated by confocal laser microscopy (TCS4D, Leica Microscopy). The isolated microvesicles were labeled by incubation with mepacrine $(0.05 \mathrm{mg} / \mathrm{ml})$ for $60 \mathrm{~min}$ at $37^{\circ} \mathrm{C}$ (Molecular Probes). Subsequently, the labeled microvesicles were added to the neutrophils obtained from the same donor and the suspensions were fixed with $2 \%$ cell fix.

\section{Flow cytometry and microvesicle sorting}

For the detection of $\mathrm{TF}$ on the platelet surface, the platelets were first incubated for $10 \mathrm{~min}$ at $37^{\circ} \mathrm{C}$ with their activators. To $100 \mu \mathrm{l}$ of cell suspension, $1 \mathrm{ml}$ of lysing solution (Becton Dickinson) was added, and the suspension was incubated for $30 \mathrm{~min}$ at room temperature. After centrifugation at $1,100 \mathrm{~g}$ for $5 \mathrm{~min}$, the supernatant was discarded and the pellet was incubated for $15 \mathrm{~min}$ in the dark with $1 \mu \mathrm{g}$ of the PE-labeled anti-TF antibody and the respective control antibody. One milliliter of PBS was added. The mixture was centrifuged for 5 min, the supernatant was discarded, and the pellet was redissolved in $500 \mu \mathrm{l}$ of PBS. The suspensions were analyzed using a FACScan flow cytometer (Becton Dickinson). Under principally identical conditions, the association of mepacrine-labeled microvesicles with the different blood components was analyzed in whole blood. For the isolation of platelet- and neutrophil-derived microvesicles, the isolated circulating microvesicles were sorted by using labeled antibodies against the platelet- and neutrophil-specific surface markers CD42b and CD66b. We were unable to obtain a pure preparation of CD14 $(+)$ microvesicles (of monocytic origin), as the CD14 $(+)$ microvesicles were also positive for CD42b. The sorting of the microvesicles was performed in a FACS-Vantage (Becton Dickinson).

\section{Western blot}

Microvesicles and platelets previously activated for $15 \mathrm{~min}$ with collagen $(8 \mu \mathrm{g} / \mathrm{ml})$ and thrombin $(0.5 \mathrm{U} / \mathrm{ml}))$ were solubilized with $1 \%$ Triton $\mathrm{X}-100$. The amount of protein was determined and extracts containing the same amount of protein were loaded onto the gel (SDS- 
PAGE with a $12 \%$ separating gel). After electroblotting, the membranes were exposed to the anti-TF antibody (VIC7) and then to a horseradish peroxidase-conjugated anti-mouse IgG.

\section{ELISA for TF}

The TF antigen content of washed microvesicles and washed blood cells and of PPP was determined using a sandwich-type ELISA with two monoclonal antibodies as described previously (18), with slight modifications (18). Briefly, microtiter plates (Maxisorp, NUNC) coated with purified anti-TF mAb VIC7 $(2.5 \mu \mathrm{g} / \mathrm{ml})$ were incubated with cell lysates or plasma diluted in sample buffer [50 mM Tris-HCl, $100 \mathrm{mM} \mathrm{NaCl}, 0.2 \%(\mathrm{v} / \mathrm{v})$ Triton X-100, 1\% BSA (w/v), $\mathrm{pH}$ 7.6] for $2 \mathrm{~h}$ at $37^{\circ} \mathrm{C}$. Twofold serial dilutions of recombinant $\mathrm{TF}$ (American Diagnostica Inc.) in sample buffer were used as standard. Thereafter, the samples were incubated with the peroxidase-labeled anti-TF mAb IIID8 for $90 \mathrm{~min}$ at $37^{\circ} \mathrm{C}$. After substrate reaction with TMB (K and $\mathrm{P}$ Laboratories) for $20 \mathrm{~min}$, the absorbance was measured at $450 \mathrm{~nm}$ with a multichannel photometer. TF values were expressed as picograms TF antigen per milligrams of total protein (microvesicles and cells) or as picograms TF per milliliter (plasma). Protein content was determined using the Micro BCA protein assay reagent kit (Pierce).

\section{Factor Xa formation}

Platelets, neutrophils, and microvesicles were isolated as described above. The isolated blood components were incubated for different time intervals in $170 \mu \mathrm{l}$ of the resuspension buffer at $37^{\circ} \mathrm{C}$. Fifty microliter portions of the suspensions were given to $100 \mu 1$ of $0.88 \mathrm{U} / \mathrm{ml}$ factor VII present in a coagulation factor concentrate (additionally containing factors II, IX, X (Beriplex $\mathrm{P} / \mathrm{N}$ 500; Aventis-Behring)), together with $50 \mu \mathrm{l}$ of a suspension containing $8 \mathrm{mM} \mathrm{CaCl}_{2}$ and 125 $\mu \mathrm{g} / \mathrm{ml}$ (final concentrations) of the chromogenic substrate S2222 (Chromogenix). After incubation for $1 \mathrm{~min}$, the increase in the optical density at $405 \mathrm{~nm}$ was registered in five subsequent $360 \mathrm{~s}$ intervals in an ELISA reader (Dynatech MR 7000). Each value was determined in triplicate. A standard curve was prepared using dilutions of tissue factor concentrates. Under all conditions analyzed, similar results for the factor $\mathrm{X}$ activation were obtained, when the isolated blood components had been washed once before the incubations in the resuspension buffer.

\section{Whole blood coagulometry}

The rates of fibrin formation and of clot growth in the whole blood were determined by computerized thrombelastography (roTEG, Dynabyte). Coagulation was started by recalcification of the citrated blood. Corn trypsin inhibitor $(30 \mu \mathrm{g} / \mathrm{ml})$ was present under all conditions in order to exclude the participation of the contact pathway of coagulation.

\section{Statistics}

The results were subjected to statistical analysis by paired $t$ test or one-way ANOVA for multiple comparisons, where appropriate. 


\section{RESULTS}

\section{TF on circulating blood microvesicles initiates coagulation}

We activated whole blood for 10 min with collagen and investigated the localization of the TF antigen by screening blood samples from three normal donors. Repeatedly, the TF antigen was detected on the surface of structures with diameters between 300-600 nm, which were surrounded by a clearly discernible bilayer (Fig. 1a). The morphology and size of the structures were indicative of microvesicles, which are known to be shedded from the plasma membrane of various cells. The TF-containing structures could represent circulating (preformed) microvesicles, or they might have been generated during the stimulation with collagen. We isolated the microvesicles from the plasma of unstimulated blood and analyzed their diameter by flow cytometry. Although most of the microvesicles represented microparticles $(>100 \mathrm{~nm})$ (Fig. $\underline{1 b}$; upper panel), also structures with diameter below $100 \mathrm{~nm}$ were observed, most probably representing exosomes (19). A similar range of microvesicle diameters was found in electron micrographs (not shown). The diameter distribution of the isolated microvesicles was similar to the one found in the whole blood (Fig. 1b; middle panel). Moreover, the expression pattern of cell-specific markers on the surface of the isolated microvesicles was comparable to the one of the whole blood-associated microvesicles (to be described elsewhere). In view of these findings, it was unlikely that the composition of the microvesicles had been altered by the isolation procedure.

After removal of the microvesicles from the plasma and readdition of the different components to obtain whole blood, it was completely devoid of the microvesicles (Fig. 1b; lower panel). In plasma selectively freed from the microvesicles, the TF concentrations were reduced by $71 \%$ (Fig. 1c). Together, the findings indicated the circulating microvesicles as the major site of the plasma-based TF. Next, we analyzed whether the preformed microvesicles contributed to the functional activation of the intravascular TF. Collagen-dependent stimulation of fibrin formation in the whole blood was suppressed by the anti-TF antibody indicating TF as an initial trigger (see Fig. 1d legend). In whole blood selectively depleted from the microvesicles, the collageninduced fibrin formation was delayed (Fig. 1d). The extent of the delay caused by the

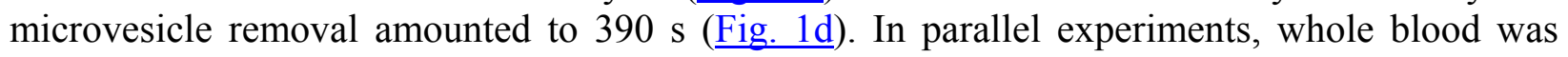
selectively depleted from the platelets. Thereby, the collagen induced fibrin formation was delayed by $330 \mathrm{~s}$. Without microvesicles and platelets, the fibrin formation was longer than 1400 $\mathrm{s}$ (means of 2 independent experiments). Without collagen stimulation, the fibrin formation also exceeded this value (Fig. 1d). Together, the data suggest an additive and comparable contribution of microvesicles and platelets to the overall fibrin generation in whole blood. After readdition of the microvesicles to the blood, the fibrin formation elicited by collagen reincreased to a similar level as observed in the control blood (not shown). In contrast, the enhanced fibrin formation in the LPS-activated blood, which depends on the expression of the monocytic TF (13), was unaffected by the removal of the microvesicles (Fig. 1d). Collagen was unable to promote the fibrin formation in the cell free plasma containing normal amounts of the microvesicles (Fig. 2a, I). Inclusion of platelets into the plasma yielding PRP resulted in some generation of fibrin (Fig. 2a, II). The stimulation was further enhanced by the additional presence of either neutrophils (Fig. 2a, IV) or monocytes (not shown). The value for the fibrin formation obtained under these conditions reached the value determined in the whole blood. Under all conditions, removal of the microvesicles attenuated the formation of fibrin (Fig. 2a, III and V). The contribution of the 
microvesicles, as calculated from the differences in fibrin formation between panels II and III, and between IV and V, was equivalent to a delay of $540 \mathrm{~s}$ in both cases. When, under the same conditions, the fibrin generation in neutrophil-enriched PPP was compared with the same parameter in neutrophil-enriched PRP, a delay of $480 \mathrm{~s}$ was found (means of 2 determinations). Together these results indicated that the collagen-induced formation of fibrin was crucially dependent on the presence of the circulating microvesicles.

The microvesicle-associated TF was functionally silent, because the short-term collagen stimulation of the ex vivo prepared microvesicles yielded no formation of factor Xa (Fig. 2b). When neutrophils were added to the microvesicle suspensions, collagen stimulated the procoagulant activity. Further addition of isolated platelets yielded a stronger increase in factor Xa formation (Fig. 2b) in support of the data in Fig. 2a. The anti-TF antibody prevented the activation, indicating that the stimulation was due to the TF-mediated cleavage of factor X (Fig. $\underline{2 b})$. To evaluate the contribution of activated factor IX for the factor Xa formation, an inhibitory antibody against factor IX was included into the microvesicle suspensions containing the platelets and neutrophils. Thereby, the factor Xa generation was reduced by $30 \%$ [from $38.9 \pm$ 9.6 (control) to $27.4 \pm 14.2$ ( + anti-IXa antibody) and $44.1 \pm 3.0 \mathrm{mU} / \mathrm{ml}$ (control antibody); means $\pm \mathrm{SD}, n=4]$. Thus, under the conditions applied, the TF/factor VIIa mediated activation of factor IXa contributed to a limited extent to the factor Xa formation. From the data of Fig. 2b, and by taking into account that the factor $\mathrm{Xa}$ formation in the microvesicle/neutrophil suspensions was completely inhibited by the anti-TF antibody (not shown), it could be calculated that $57 \%$ of the total $\mathrm{TF}$-induced factor $\mathrm{X}$ cleavage (as determined with all components) was related to the presence of the platelets. The remaining TF-dependent factor Xa formation can be accounted for by the microvesicle TF (see below and Discussion). Elevated numbers of circulating microvesicles as observed under several pathological conditions are often associated with a higher thrombotic risk (see Introduction). Increasing the microvesicle number up to 10fold without altering the concentrations of the other blood components, drastically stimulated the factor Xa formation (Fig. 2c). This was seen in the presence of the neutrophils and was more pronounced with neutrophils plus platelets. Under the latter conditions, the amount of factor Xa formed in the presence of ten-fold elevated microvesicles $(76.5 \mathrm{mU} / \mathrm{ml}$; Fig. 2c) substantially exceeded the value to be calculated from the addition of the single effect obtained with microvesicles and neutrophils $(25.8 \mathrm{mU} / \mathrm{ml}$; Fig. 2c) plus the one seen with platelets and neutrophils (in the absence of microvesicles $[11.3 \pm 2.4 \mathrm{mU} / \mathrm{ml}$ (means of 5 determinations \pm $\mathrm{SD})$; see Discussion].

\section{Intraplatelet localization and activation- induced exposure of TF}

Substantial fractions of the microvesicles circulating in human blood are derived from the platelets $(17,20)$. We isolated the circulating microvesicles of platelet origin by sorting the microvesicles with the use of an antibody against the platelet-specific surface protein CD42b (Fig. 3a, top panel). In the CD42b positive microvesicles, full-length $\mathrm{TF}$ was detected by Western blotting (Fig. 3a, bottom panel). Microvesicles recovered from supernatants of platelets stimulated in vitro with collagen and thrombin also contained TF. Moreover, TF was also detected in the activated platelets themselves (Fig. 3a, bottom panel). However, when we separated the microvesicles of neutrophil origin from the total pool of circulating microvesicles by the use of an antibody against the neutrophil surface marker CD66b, no TF protein was found. In addition, no TF could be detected in the microvesicles derived from the in vitro stimulated 
neutrophils (Fig. 3a, top panel). The TF contents of the platelet microparticles amounted to 27 $\mathrm{pg} / \mathrm{mg}$ protein, as determined by double sandwich ELISA (Fig. 3b). Again, no TF was detected in the neutrophil microvesicles. As a comparison, the TF contents of the isolated parent blood cells are shown in Fig. 3c. TF was present in the platelets but not in the neutrophils. By determining the protein contents of the microvesicles released from the platelets during the 15 min activation period with collagen and thrombin, we calculated that $5.9 \%$ of the total platelet TF hade been shedded in association with the microvesicles. Moreover, assuming a hematocrit of $50 \%$ and a platelet count of $3 \times 10^{8} / \mathrm{ml}$ blood, it could be calculated on the basis of the data from Figs. $1 \mathrm{c}$ and $\underline{3 \mathrm{~b}}$ that in $1 \mathrm{ml}$ of human blood, $30 \mathrm{pg}$ and $110 \mathrm{pg}$ of TF will be associated with the platelets and the plasma compartment, respectively.

The intracellular location of TF was analyzed in the resting platelets. In thin sections of the platelets, TF was found to be mainly located in the $\alpha$-granules as well as in the open canalicular system (Fig. 4a-d). Within the $\alpha$-granules, TF was associated with the membrane (Fig. 4a), and, in addition, with the matrix of the granules (Fig. 4c-d). Part of the platelet TF was detected in the open canalicular system (Fig. 4b). TF was completely absent from the dense granules, the mitochondria, and the platelet cytoplasma. Occasionally, TF was also located on the platelet cell surface (Fig. 4a). No labeling was seen without the primary antibodies. Moreover, no TF antigen could be detected in thin sections of bone marrow derived megakaryocytes (not shown). By the use of flow cytometry, TF was barely detectable on the surface of the isolated resting platelets. However, the protein was presented on the plasma membrane after stimulation with collagen (Fig. 4e). Thrombin activation also elicited the exposure of TF on the cell membrane, whereas ADP was ineffective (Fig. 4e). In summary, TF was found to be stored in the $\alpha$-granules and in the open canalicular system of the resting platelets. Stimulation by strong agonists led to the rapid presentation of TF on the platelet surface. Together, the findings demonstrated that the preformed TF stored intracellularly in the platelets was rapidly exposed on the surface and secreted in association with microvesicles.

\section{Cell adhesions and reactive oxygen species promote activation of intravascular TF}

To further substantiate that the microvesicles and activated platelets provided TF competent to initiate the coagulation process, the blood components (ex vivo and in vitro isolated platelet microvesicles, platelets, and neutrophils) were selectively precoated with the anti-TF antibody. Precoating of the microvesicles with the anti-TF antibody substantially decreased the formation of factor Xa (Fig. 5). Selective precoating of the neutrophils barely altered the cleavage of factor $\mathrm{X}$, whereas the procoagulant activity was clearly lowered in the presence of the precoated platelets (Fig. 5). Together, these results indicated that the circulating microvesicles as well as the platelets themselves are major sites of functionally active TF in the blood.

To identify the cellular components of the blood interacting with the microvesicles, the native circulating microvesicles were substituted by mepacrine-labeled microvesicles. Then, after a short stimulation with collagen, the associations of the microvesicles with the different blood cells were analyzed. Collagen promoted the adhesion of the microvesicles to the neutrophils and, to a somewhat lesser extent, to the monocytes (Fig. 6a). Collagen was unable to elicit the association of the microvesicles with the platelets. The strong association of the circulating microvesicles with the neutrophils was confirmed in confocal laser scan experiments with the isolated blood cells. In those experiments, the surface association of the labeled microvesicles to 
the neutrophils was diminished by the anti-P-selectin, the anti-PSGL-antibodies, and the antiCD18-antibodies (not shown). The antibodies disrupting the adhesion of platelets and microvesicles to the neutrophils inhibited the TF activity of the blood cell suspensions (Fig. 6b). Thus activation of the intravascular TF required the close association of the microvesicles and platelets with the neutrophils.

The importance of the neutrophils for the activation of the intravascular TF led us to investigate the participation of the reactive oxygen species secreted by the neutrophils (hydrogen peroxide, superoxide anion). Addition of catalase, which degrades hydrogen peroxide, substantially suppressed the TF activity of the microvesicle suspensions (Fig. 6c). Degradation of superoxide anion by superoxide dismutase (SOD) also blocked the procoagulant activity. Catalase and SOD markedly lowered the TF-dependent fibrin formation in whole blood stimulated by collagen (Fig. $\underline{6 c)}$. In contrast, the fibrin formation elicited by LPS was barely affected by the two enzymes. The results pointed to a major role for the neutrophil reactive oxygen species as activators of the intravascular TF. Due to the requirement for platelet-neutrophil adhesions for the activation of $\mathrm{TF}$, we evaluated whether the oxygen radicals would enhance the adhesion between the two blood components. However, catalase and SOD did not reduce the adhesion of the platelets to the neutrophils, as determined by the number of $\mathrm{CD} 42 \mathrm{~b}$ positive neutrophils in the collagen stimulated blood (not shown). Together, our results suggested that the TF activity of activated platelets and microvesicles is markedly enhanced by neutrophil adhesion molecules and secretion products.

\section{DISCUSSION}

Our data demonstrate that TF present on circulating microvesicles and preformed TF exposed on the surface of the activated platelets are the major physiological effectors of the initiation of coagulation in blood. Under all conditions investigated, in whole blood, in plasma, and in suspensions of isolated blood components, microvesicles and activated platelets were found to be required to elicit the TF-dependent activation of coagulation. In line with this view, without platelets and microvesicles, no TF-dependent fibrin formation was measurable. In both blood components, TF was detected by different methods. Importantly, circulating microvesicles and activated platelets contributed to a similar extent and additively to the overall initiation of intravascular coagulation, as indicated by whole blood and single component protocols.

Our study provides the first evidence for the localization of TF in platelets. In nonactivated platelets, TF is mainly located in the open canalicular system and in the $\alpha$-granules. This location pattern clearly indicates that $\mathrm{TF}$ is incorporated into the platelet interior and not just associated with the cell membrane. Fusion of the granule membrane with the cell membrane is expected to expose TF on the platelet surface in a regulated step during platelet activation. Because TF could not be detected in the megakaryocytes, platelet TF is likely to be derived from extracellular donors. Other intraplatelet procoagulant proteins such as fibrinogen and factor $\mathrm{V}$ are indeed known to be internalized into the megakaryocytes and platelets $(21,22)$. Thus platelets represent a storage pool for the intravascular TF.

The presence of functionally active TF in circulating microvesicles and platelets could explain why substantial blood-based TF activity is retained after the selective depletion of leukocytes from blood (23). Microvesicles are shedded by various blood cells in response to activation, 
maturation, mechanical stress, and other stimuli. Their number is increased under conditions associated with an enhanced thrombotic risk, such as acute coronary disease, heparin-induced thrombocytopenia, and sepsis $(17,24-26)$. Our findings suggest that the microvesicle TF contributes to a relevant extent to this prothrombotic state. In line with this conclusion, high plasma TF contents have been observed under pathological conditions predisposing for thrombosis (7-9). Indeed, when present in normal concentrations, the microvesicle TF was found to account already for about one-half of the overall TF-mediated activation of coagulation. This was evident from the collagen-induced increases in the fibrin formation in whole blood and the enhancement of the TF activity in suspensions of single blood components. Under whole blood conditions, collagen elicits the rapid shedding of microvesicles from the activated platelets, resulting in the dissemination of procoagulant phospholipids such as phosphatidylserine. Thus, the release of procoagulant phospholipids is expected to contribute to the increased fibrin formation on stimulation of whole blood with collagen.

The procoagulant activity was dramatically enhanced, when the microvesicle numbers were increased up to 10-fold, encompassing the range of increases in microvesicles as determined under conditions favoring thrombogenesis $(17,20,24-26)$. The enhancement was particularly prominent, when platelets were added to the neutrophil suspensions supplemented with high amounts of microvesicles. Activated platelets are known to enhance the secretion of reactive oxygen species by the neutrophils (27). Because reactive oxygen species were found to activate the microvesicle TF, this mechanism could be responsible for the overadditive effect elicited by the platelets. On activation of whole blood with collagen, the circulating microvesicles were observed to adhere to the neutrophils and monocytes and disruption of the platelet-neutrophil adhesions diminished the intravascular TF activity. Adhesion of platelets to neutrophils has been proposed to result in the formation of a restricted microenvironment, with high local concentrations of the neutrophil secretion products (28). Although the reactive oxygen species are needed to activate the intravascular TF, they apparently act in concert with the neutrophil proteases, which, in parallel, might inactivate tissue factor pathway inhibitor (14). We could exclude that the effect of the reactive oxygen species was due to their potential proadhesive influence. Alternatively, the oxygen species could promote the activation of latent (encrypted) $\mathrm{TF}$, as recently proposed (29).

Platelets are apparently the early targets of collagen under in vivo conditions, because in GPVI (collagen receptor)-deficient mice, thrombosis is considerably delayed after injection of the matrix protein (30). Furthermore, mice deficient in the platelet secretion product Gas6 (31) are also resistant to the formation of venous thrombemboli in response to collagen. According to our findings, it is likely that the fibrin formation elicited by collagen in vivo is also triggered by the microvesicle and platelet TF. Our findings could substantially advance our understanding of the pathogenesis of venous thrombosis. TF has been proposed as a major trigger for pathological fibrin formation in the venous system (3). Venous thrombosis often develops on the injured endothelium, without exposure of subendothelial structures to the flowing blood (32). Moreover, fibrin deposition is particularly prominent in venous thrombi. The adhesion of neutrophils, platelets, and microvesicles to the endothelium, which was already shown to favor the generation of thrombi (33), will facilitate the local accumulation and activation of the intravascular TF.

Although TF expressed in the adventitial layer of the vessel wall represents the major initiator of physiological coagulation, some observations point to a principal role for the intravascular TF in 
the overall hemostatic process. The platelet layer adhering to the subendothelial collagen within seconds after the vessel wall rupture will indeed impede the diffusion of factor Xa from the TF/VIIa complex of the vessel wall to the growing thrombus. Because the thrombus is growing toward the direction of the lumen, it is unlikely that substantial amounts of adventitial factor Xa will reach those platelets being recruited to the initial platelet layer. On the other hand, the factor Xa generated by the TF/VIIa complex of the platelets and microvesicles can be directly channeled into the prothrombinase complex being assembled on the surface of the same blood particles, without substantial loss in quantity or time. In conclusion, the intravascular $\mathrm{TF}$ apparently enables the formation of fibrin at the site of the growing thrombus. Under those conditions, the whole coagulation process will take place on the surface of the blood cells, in particular on microvesicles and platelets.

\section{ACKNOWLEDGMENTS}

This study was supported by grants of the Deutsche Forschungsgemeinschaft to B.E.

\section{REFERENCES}

1. Dahlback, B. Blood coagulation. (2000) Lancet 355, 1627-1632

2. Rauch, U., Osende, J. I., Fuster, V., Badimon, J. J., Fayad, Z., and Chesebro, J. H. (2001) Thrombus formation on atherosclerotic plaques: pathogenesis and clinical consequences. Ann. Intern. Med. 134, 224-238

3. Lawson, C. A., Yan, S. D., Yan, S. F., Liao, H., Zhou, Y. S., Sobel, J., Kisiel, W., Stern, D. M., and Pinsky, D. J. (1997) Monocytes and tissue factor promote thrombosis in a murine model of oxygen deprivation. J. Clin. Invest. 99, 1729-1738

4. Thiruvikraman, S. V., Guha, A., Roboz, J., Taubman, M. B., Nemerson, Y., and Fallon, J. T. (1996) In situ localization of tissue factor in human atherosclerotic plaques by binding of digoxigenin-labeled factors VIIa and X. Lab. Invest. 75, 451-461

5. Toschi V., Gallo R., Lettino M., Fallon J. T., Gertz S. D., Fernandez-Ortiz A., Chesebro J. H., Badimon L., Nemerson Y., Fuster V., and Badimon, J. J. (1997) Tissue factor modulates the thrombogenicity of human atherosclerotic plaques. Circulation 95, 594-599

6. Kaikita, K., Ogawa, H., Yasue, H., Takeya, M., Takahashi, K., Saito, T., Hayasaki, K., Horiuchi, K., Takizawa, A., Kamikubo, Y., Soejima, H., and Yasue, H. (1997) Tissue factor expression on macrophages in coronary plaques in patients with unstable angina. Arterioscler. Thromb. Vasc. Biol. 17, 2232-2237

7. Suefuji, H., Ogawa, H., Yasue, H., Kaikita, K., Soejima, H., Motoyama, T., Mizuno, Y., Oshima, S., Saito, T., Tsuji, I., Kumeda, K., Kamikubo, Y., and Nakamura, S. (1997) Increased plasma tissue factor levels in acute myocardial infarction. Am. Heart J. 134, 253-259

8. Misumi, K., Ogawa, H., Yasue, H., Soejima, H., Suefuji, H., Nishiyama, K., Takazoe, K., Kugiyama, K., Tsuji, I., Kumeda, K., and Nakamura, S. (1998) Comparison of plasma tissue factor levels in unstable and stable angina pectoris. Am. J. Cardiol. 81, 22-26 
9. $\quad$ Soejima, H., Ogawa, H., Yasue, H., Kaikita, K., Nishiyama, K., Misumi, K., Takazoe, K., Miyao, Y., Yoshimura, M., Kugiyama, K., Nakamura, S., Tsuji, I., and Kumeda, K. (1999) Heightened tissue factor associated with tissue factor pathway inhibitor and prognosis in patients with unstable angina. Circulation 99, 2908-2913

10. Nemerson, Y. (2000) Tissue factor in neutrophils. Thromb. Haemost. 83, 802

11. Ozcan, M., Morton, C. T., Solovey, A., Dandelet, L., Bach, R. R., Hebbel, R. P., Slungaard, A., and Key, N. S. (2001) Whole blood tissue factor procoagulant activity remains detectable during severe aplasia following bone marrow and peripheral blood stem cell transplantation. Thromb. Haemost. 85, 250-255

12. Bouchard, B. A., and Tracy, P. B. (2001) Platelets, leukocytes, and coagulation. Curr. Opin. Hematol. 8, 263-269

13. Osterud, B. (1998) Tissue factor expression by monocytes: regulation and pathophysiological roles. Blood Coagul. Fibrinolysis 9, Suppl 1, S9-14

14. Zillmann, A., Luther, T., Muller, I., Kotzsch, M., Spannagl, M., Kauke, T., Oelschlagel, U., Zahler, S., and Engelmann, B. (2001) Platelet-associated tissue factor contributes to the collagen-triggered activation of blood coagulation. Biochem. Biophys. Res. Commun. 281, 603609

15. Rauch, U., Bonderman, D., Bohrmann, B., Badimon, J. J., Himber, J., Riederer, M. A., and Nemerson, Y. (2000) Transfer of tissue factor from leukocytes to platelets is mediated by CD15 and tissue factor. Blood 96, 170-175

16. Randolph, G. J., Luther, T., Albrecht, S., Magdolen, V., and Muller, W. A. (1998) Role of tissue factor in adhesion of mononuclear phagocytes to and trafficking through endothelium in vitro. Blood 92, 4167-4177

17. Nieuwland, R., Berckmans, R. J., McGregor, S., Boing, A. N., Romijn, F. P., Westendorp, R. G., Hack, C. E., and Sturk, A. (2000) Cellular origin and procoagulant properties of microparticles in meningococcal sepsis. Blood 95, 930-935

18. Albrecht, S., Luther, T., Grossmann, H., Flossel, C., Kotzsch, M., and Muller, M. (1992) An ELISA for tissue factor using monoclonal antibodies. Blood Coagul. Fibrinolysis 3, 263-270

19. Denzer, K., Kleijmeer, M. J., Heijnen, H. F., Stoorvogel, W., and Geuze, H. J. (2000) Exosome: from internal vesicle of the multivesicular body to intercellular signaling device. $J$. Cell Sci. 19, 3365-3374

20. Joop, K., Berckmans, R. J., Nieuwland, R., Berkhout, J., Romijn, F. P., Hack, C. E., and Sturk, A. (2001) Microparticles from patients with multiple organ dysfunction syndrome and sepsis support coagulation through multiple mechanisms. Thromb. Haemost. 85, 810-812 
21. Handagama, P., Rappolee, D. A., Werb, Z., Levin, J., and Bainton, D. F. (1990) Platelet alpha-granule fibrinogen, albumin, and immunoglobulin $\mathrm{G}$ are not synthesized by rat and mouse megakaryocytes. J. Clin. Invest. 86, 1364-1368

22. Camire, R. M., Pollak, E. S., Kaushansky, K., and Tracy, P. B. (1998) Secretable human platelet-derived factor V originates from the plasma pool. Blood 92, 3035-3041

23. Ozcan, M., Morton, C. T., Solovey, A., Dandelet, L., Bach, R. R., Hebbel, R. P., Slungaard, A., and Key, N. S. (2001) Whole blood tissue factor procoagulant activity remains detectable during severe aplasia following bone marrow and peripheral blood stem cell transplantation. Thromb. Haemost. 85, 250-255

24. Mallat, Z., Benamer, H., Hugel, B., Benessiano, J., Steg, P. G., Freyssinet, J. M., and Tedgui, A. (2000) Elevated levels of shed membrane microparticles with procoagulant potential in the peripheral circulating blood of patients with acute coronary syndromes. Circulation 101, $841-843$

25. Nieuwland, R., Berckmans, R. J., Rotteveel-Eijkman, R. C., Maquelin, K. N., Roozendaal, K. J., Jansen, P. G., ten Have, K., Eijsman, L., Hack, C. E., and Sturk, A. (1997) Cell-derived microparticles generated in patients during cardiopulmonary bypass are highly procoagulant. Circulation 96, 3534-3541

26. Hughes, M., Hayward, C. P., Warkentin, T. E., Horsewood, P., Chorneyko, K. A., and Kelton, J. G. (2000) Morphological analysis of microparticle generation in heparin-induced thrombocytopenia. Blood 96, 188-194

27. Nagata, K., Tsuji, T., Todoroki, N., Katagiri, Y., Tanoue, K., Yamazaki, H., Hanai, N., and Irimura, T. (1993) Activated platelets induce superoxide anion release by monocytes and neutrophils through P-selectin (CD62). J. Immunol. 151, 3267-3273

28. Evangelista, V., Rajtar, G., de Gaetano, G., White, J. G., and Cerletti, C. (1991) Platelet activation by fMLP-stimulated polymorphonuclear leukocytes: the activity of cathepsin G is not prevented by antiproteinases. Blood 77, 2379-2388

29. Penn, M. S., Patel, C. V., Cui, M. Z., DiCorleto, P. E., and Chisolm, G. M. (1999) LDL increases inactive tissue factor on vascular smooth muscle cell surfaces: hydrogen peroxide activates latent cell surface tissue factor. Circulation 99, 1753-1759

30. Nieswandt, B., Schulte, V., Bergmeier, W., Mokhtari-Nejad, R., Rackebrandt, K., Cazenave, J. P., Ohlmann, P., Gachet, C., and Zirngibl, H. (2001) Long-term antithrombotic protection by in vivo depletion of platelet glycoprotein VI in mice. J. Exp. Med. 193, 459-469

31. Angelillo-Scherrer, A., de Frutos, P., Aparicio, C., Melis, E., Savi, P., Lupu, F., Arnout, J., Dewerchin, M., Hoylaerts, M., Herbert, J., Collen, D., Dahlback, B., and Carmeliet, P. (2001) Deficiency or inhibition of Gas6 causes platelet dysfunction and protects mice against thrombosis. Nat. Med. 7, 215-221 
32. Line, B. R. (2001) Pathophysiology and diagnosis of deep venous thrombosis. Semin. Nucleic Med. 31, 90-101

33. May, A. E., Neumann, F. J., and Preissner, K. T. (1999) The relevance of blood cellvessel wall adhesive interactions for vascular thrombotic disease. Thromb. Haemost. 82, 962970

Received July 10, 2002; accepted November 20, 2002. 
Fig. 1

a

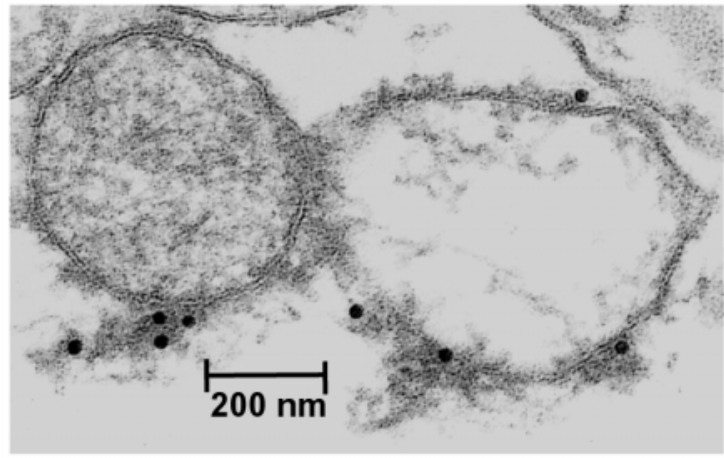

b
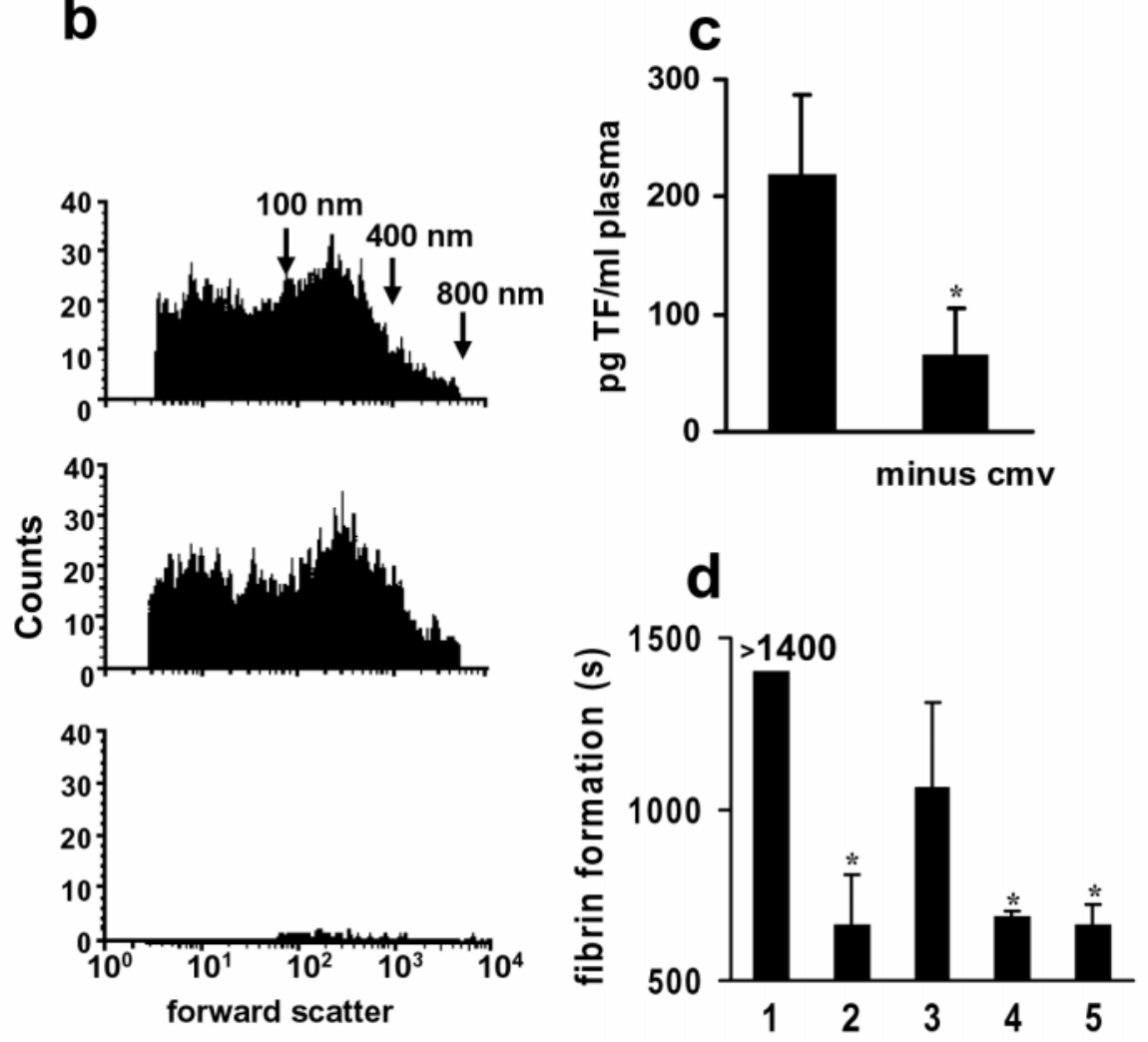

Figure 1. Microvesicles of human blood express TF. a) Electronmicrographs of particles representing microvesicles in whole blood stimulated with collagen $\left(12 \mu \mathrm{g} / \mathrm{ml} ; 10 \mathrm{~min}, 37^{\circ} \mathrm{C}\right)$. TF antigen is detected by immunoelectronmicroscopy on and close to the membrane of microvesicles. b) Size of blood microvesicles as determined by flow cytometry.

Microvesicles were isolated from whole blood as described in Methods. Diameters of isolated circulating microvesicles (top panel) were estimated by using lipid vesicles of defined size as prepared by an extrusion technique (arrows). An identical distribution was seen in the whole blood (middle panel). Effective removal of the microvesicles from the blood by high speed centrifugation of plasma (bottom panel). c) Removal of circulating microvesicles reduces the plasma TF contents. Plasma was depleted from microvesicles by sequential centrifugation steps. TF contents were estimated by a double sandwich ELISA by the use of 2 different antibodies directed against the extracellular domain of TF. Cmv, circulating microvesicles. Results are means $\pm \mathrm{SD}(n=10)$. ${ }^{*} P<0.05$ (vs. native plasma). d) Collagen-induced fibrin formation is delayed after removal of microvesicles from blood. Collagen-elicited fibrin formation in whole blood was prevented by the anti-TF antibody $(20 \mu \mathrm{g} / \mathrm{ml})$, confirming its dependence on the intravascular TF (from $840 \mathrm{~s}(12 \mu \mathrm{g} / \mathrm{ml}$ collagen) to $>1,600 \mathrm{~s}$ (plus the anti-TF antibody VIC7); 1, control; 2, collagen; 3, collagen, blood without microvesicles; 4, LPS (10 $\mu \mathrm{g} / \mathrm{ml} ; 90$ min preincubation); 5, LPS, without microvesicles. Results are means \pm SD $(n=6)$. * $P<0.05$ (vs. control). 
Fig. 2

a
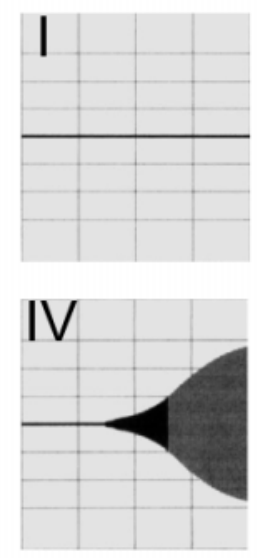
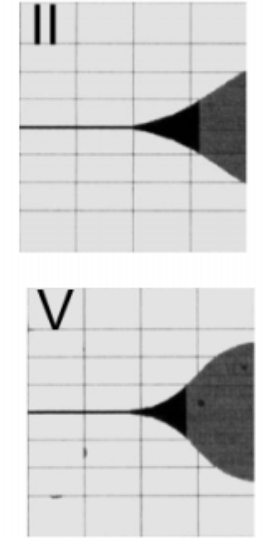
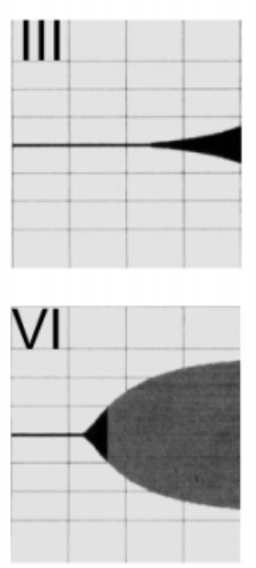

C

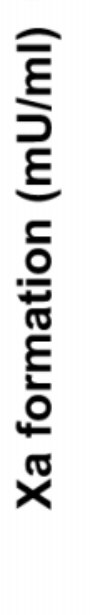

b

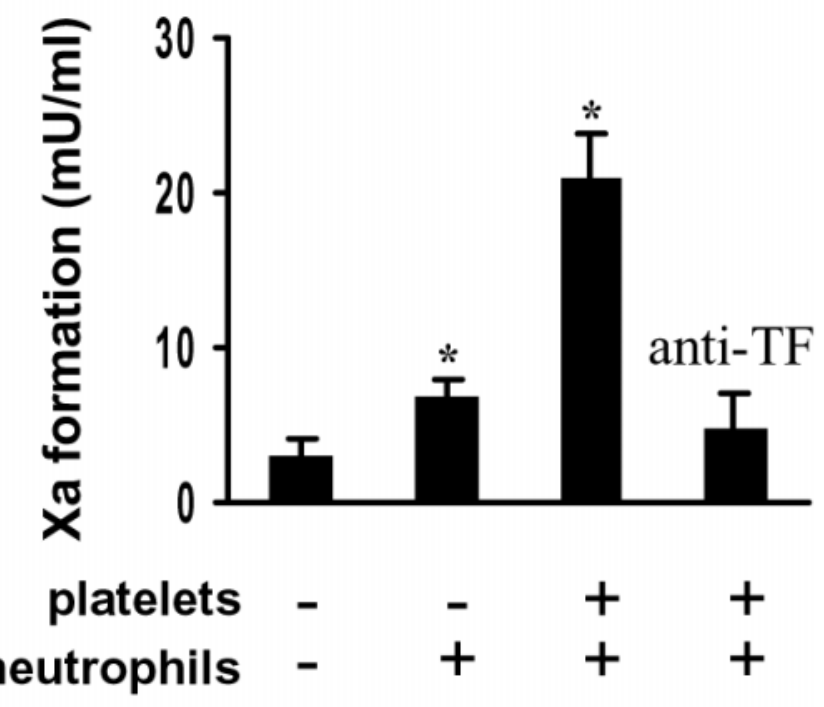

Figure 2. Microvesicle TF promotes fibrin formation. a) Microvesicles and platelets are required for collagen-induced fibrin formation in whole blood. Thrombelastograms of whole blood stimulated with collagen (total running time $=45$ min). The fibrin formation represents the distance between the start and the broadening of the amplitude. I, PPP; II PRP; III, PRP without microvesicles; IV, PRP and neutrophils; V, PRP and neutrophils without microvesicles; VI, whole blood. b) Platelets promote the TF-dependent factor Xa formation in microvesicle suspensions. To isolated circulating microvesicles $\left(3 \times 10^{4}\right.$, dissolved in $170 \mu \mathrm{l}$ of resuspension buffer), platelets $\left(2 \times 10^{7}\right)$ and neutrophils $\left(2 \times 10^{6}\right)$ were added as indicated. All samples were stimulated with collagen $\left(5 \mathrm{~min}, 37^{\circ} \mathrm{C}\right)$. Factor $\mathrm{Xa}$ formation was measured by a chromogenic substrate. Results are means $\pm \mathrm{SD}(n=6) .{ }^{*} P<0.05$ (vs. microvesicles alone). c) Increasing the microvesicle number at a constant amount of blood cells enhances factor Xa formation; $\bullet$, microvesicles; $\mathbf{-}$, microvesicles plus neutrophils; $\mathbf{\Lambda}$, microvesicles plus neutrophils and platelets. Values given on $y$-axis refer to the increase in microvesicle number compared with the basal number of microvesicles ( $3 \times 10^{4}$, set as "1"). Representative experiment of a total of 5 experiments. Neutrophils and platelets were present in similar amounts as in $\mathbf{b}$. 
Fig. 3
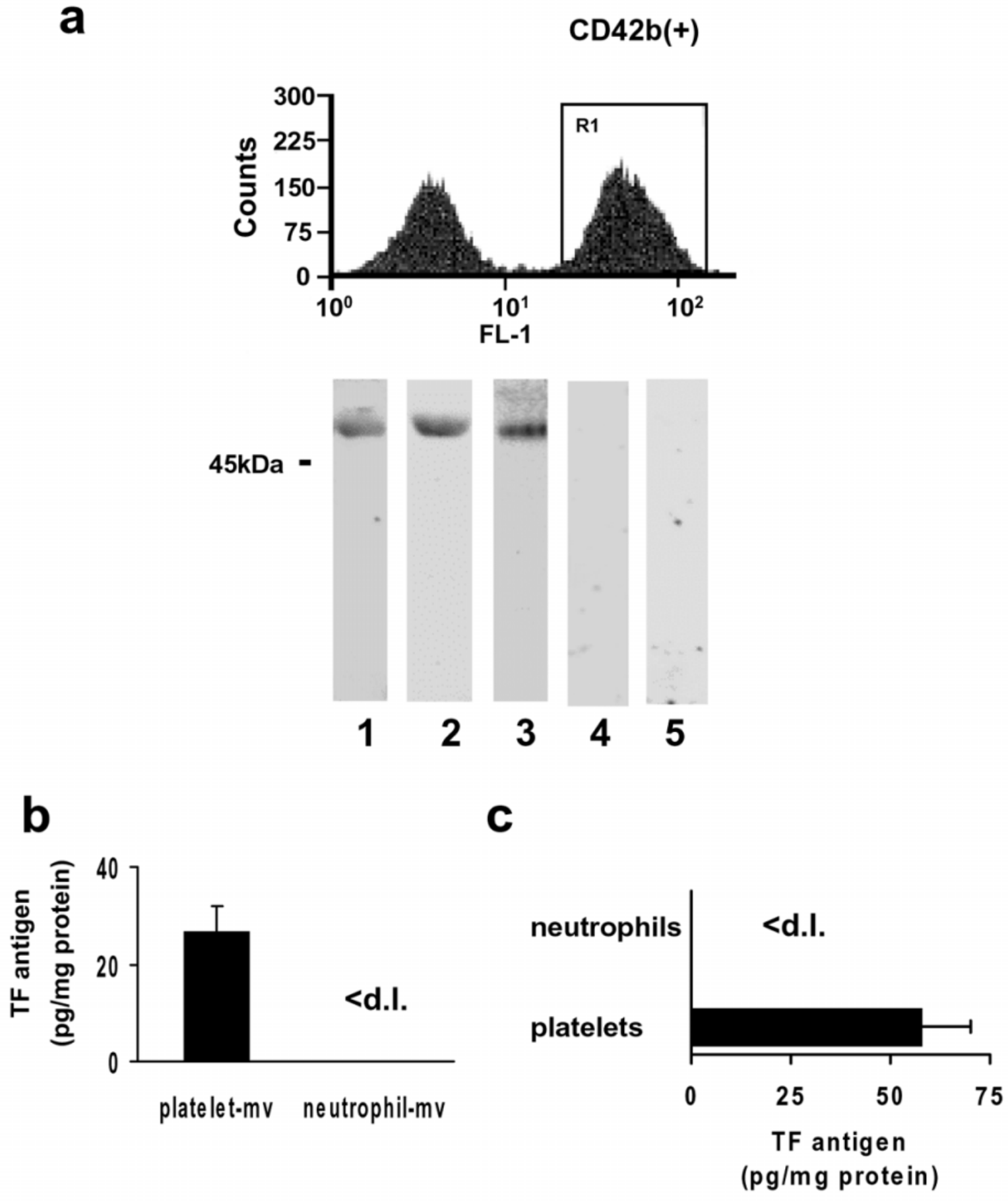

Figure 3. Platelet microvesicles and platelets are major sources of intravascular TF. a) Top panel: separation of $\mathrm{CD} 42 \mathrm{~b}$ positive microvesicles from total circulating microvesicles. After isolation of the total pool of circulating microvesicles, the microvesicles were sorted by the use of cell specific surface markers. Bottom panel: detection of fulllength TF ( $47 \mathrm{kDa})$ by Western blotting in CD42b positive microvesicles (lane 1), in vitro generated platelet microvesicles (lane 2), and in activated platelets (lane 3). CD66b positive circulating microvesicles (lane 4) and microvesicles obtained from in vitro activated neutrophils (lane 5) are devoid of TF. Identical amounts of protein were loaded onto gel in all cases. b, c) TF contents of microvesicles shedded from activated platelets and neutrophils (b) and of the respective parent cells of unstimulated blood (c). d.1., Detection limit. Results are means \pm SD ( $n=3-5)$. 
Fig. 4
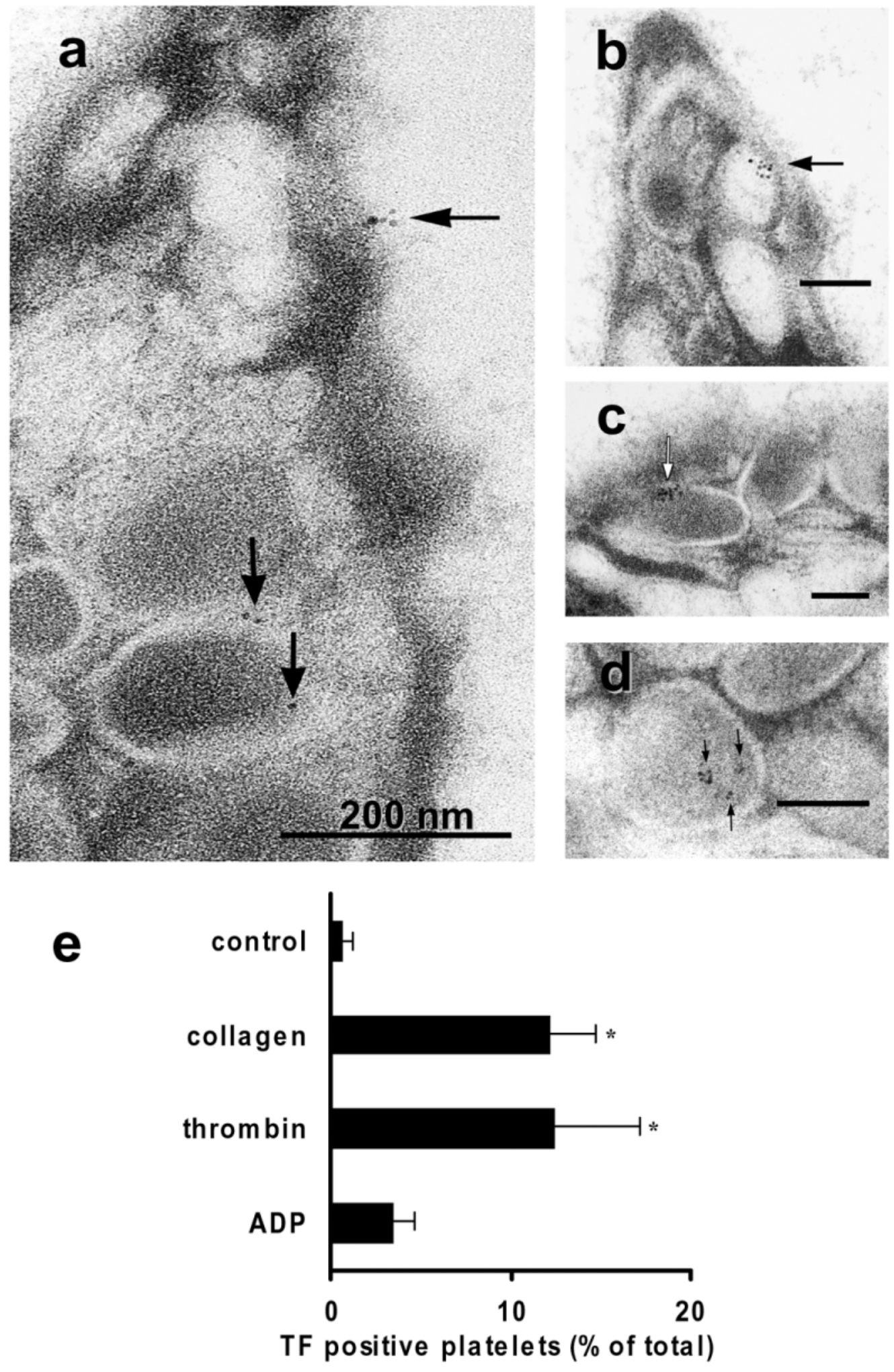

Figure 4. Intraplatelet localization of TF. a) Immunoelectronmicrographs showing TF antigen in the membrane of $\alpha$ granules and on the cell surface. b) Localization of TF in the open canalicular system of resting platelets. c, d) TF antigen in the matrix and membrane of $\alpha$-granules. e) Presentation of TF on the surface of activated platelets. Isolated platelets were stimulated with collagen $(12 \mu \mathrm{g} / \mathrm{ml} ; 10 \mathrm{~min})$, thrombin $(0.5 \mathrm{U} / \mathrm{ml})$, and ADP $(10 \mu \mathrm{M})$, and surface presentation of TF was analyzed by flow cytometry. Results are means \pm SD $(n=4) . * P<0.05$ (vs. control). 
Fig. 5

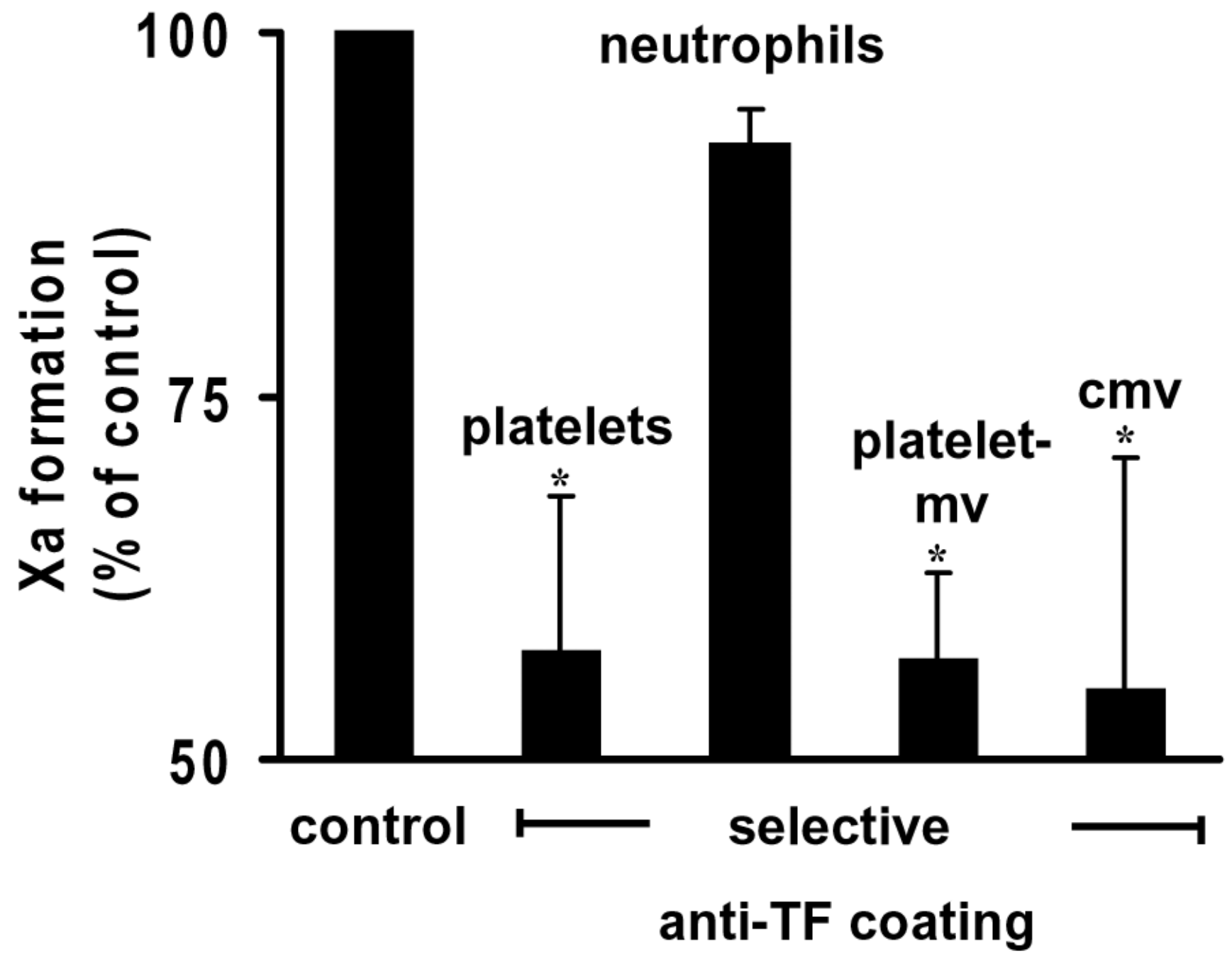

Figure 5. Functionally active TF on platelets and their microvesicles. Precoating of microvesicles and platelets with anti-TF antibody prevents factor Xa cleavage. Microvesicles (either in vitro produced by platelets or circulating microvesicles, as indicated), neutrophils, and platelets were separately precoated for 20 min with anti-TF antibody (VIC7, $10 \mu \mathrm{g} / \mathrm{ml})$. Precoated blood components were washed and added to the other nonprecoated blood components. Thereafter, the suspensions containing the precoated and nonprecoated components were stimulated for 5 min with collagen. The quantities of platelets, neutrophils, and microvesicles present in the resuspension buffer were $2 \times 10^{7}, 2 \times 10^{6}$, and $3 \times 10^{4}$. Control value was determined with all components in the absence of antibody. Results are means $\pm \operatorname{SD}(n=4)$. $* P<0.05$ (vs. control). 
Fig. 6
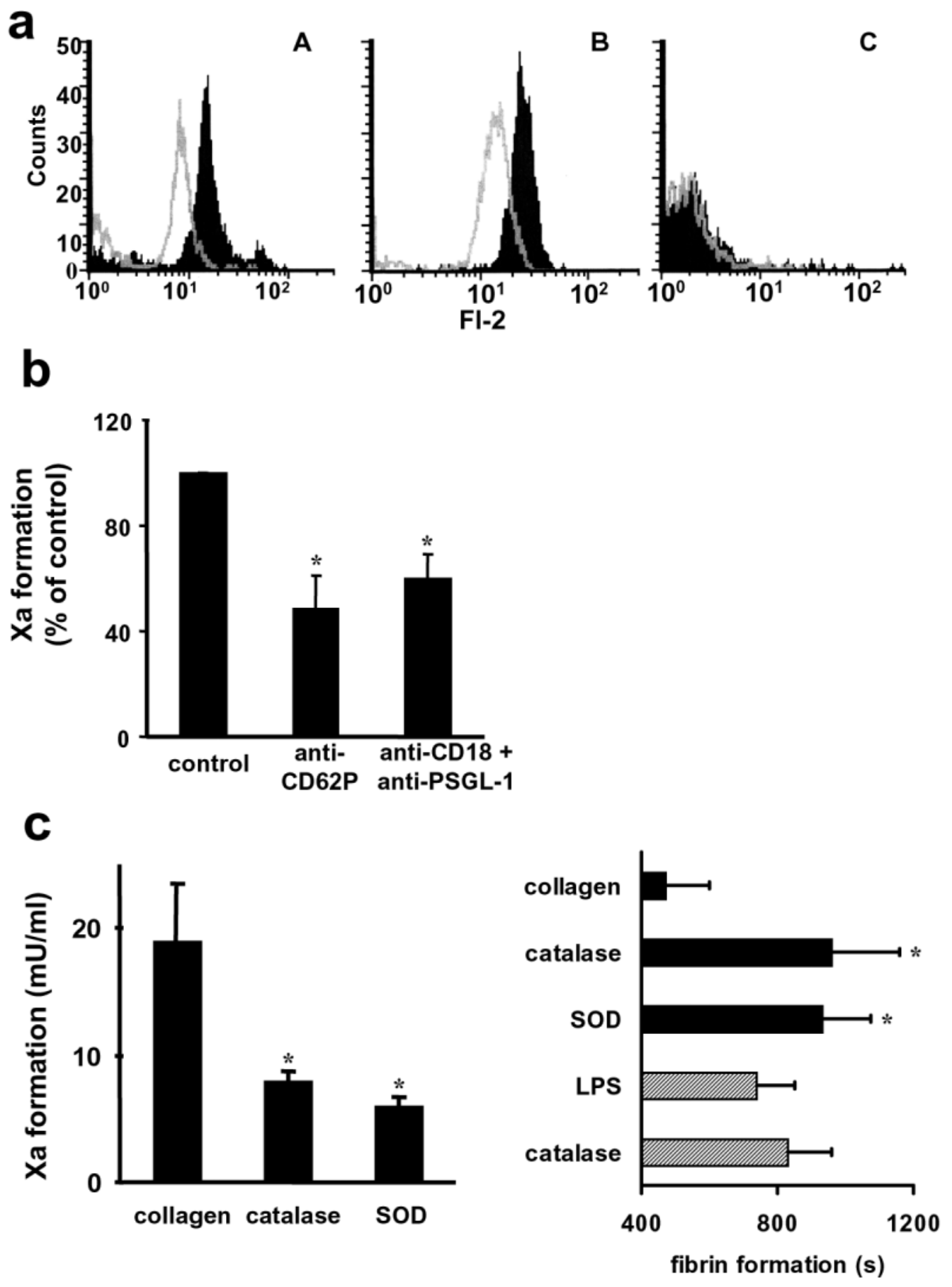

Figure 6. Cell adhesions and reactive oxygen species activate intravascular TF. a) Microvesicles adhere to neutrophils and monocytes in collagen-stimulated blood. Blood containing mepacrin-labeled microvesicles was stimulated with collagen. Blood cells were identified by their forward and sideward scatter characteristics. A, monocytes; B, neutrophils; C, platelets. Empty curves, control; filled curves, collagen. b) Antibodies disrupting platelet-neutrophil adhesions reduce the intravascular TF activity. Suspensions of platelets and neutrophils supplemented with circulating microvesicles were stimulated with collagen. The quantities of platelets, neutrophils, and microvesicles present in the resuspension buffer were $2 \times 10^{7}, 2 \times 10^{6}$, and $3 \times 10^{4}$. The antibodies were added at $10 \mu \mathrm{g} / \mathrm{ml}$. Results are means $\pm \mathrm{SD}$ $(n=4) . * P<0.05$ (vs. control). c) Catalase and SOD inhibit the intravascular TF activity. The TF activity of platelets plus platelet microvesicles (in the presence of neutrophils; left panel) and TF-dependent fibrin formation in whole blood (right panel) are suppressed by catalase $(250 \mathrm{U} / \mathrm{ml})$ and SOD $(12 \mu \mathrm{g} / \mathrm{ml})$. Results are means \pm SD $(n=5) . * P<0.05$ (vs. collagen). 